\title{
T Regulatory Cells and Transplantation Tolerance
}

\author{
Vijay S. Gorantla, MD, Stefan Schneeberger, MD, Gerald Brandacher, MD, Robert Sucher, \\ MD, Dong Zhang, MD, PhD, WP, Andrew Lee, MD, and Xin Xiao Zheng, MD \\ Division of Plastic Surgery, Department of Surgery, University of Pittsburgh School of Medicine
}

\begin{abstract}
Despite the development of successful immunosuppression protocols and tremendous improvement in short-term graft survival rates, the problem of chronic graft loss remains the bane of clinical transplantation. The induction and maintenance of transplantation tolerance is the "Holy grail" of transplantation.
\end{abstract}

The recent identification and characterization of regulatory $T$ cells ( $T$ regs) has opened up exciting opportunities for tolerance induction, immunotherapy and immunomodulation in transplantation. This review focuses on current understanding of regulatory $\mathrm{T}$ cells and their role in transplantation tolerance.

\section{Introduction}

Groundbreaking advancements in immunology and immunosuppressive drug therapies during the last four decades have enabled widespread application of solid organ and hematopoietic stem cell transplantation in the life saving treatment of a spectrum of diseases. Despite the development of successful immunosuppression protocols and tremendous improvement in short-term graft survival rates, the problem of chronic graft loss remains the bane of clinical transplantation $(1,2)$. Further, none of the current immunosuppressive strategies culminate in true graft tolerance. Most importantly, the toxicity and complication profile of standard immunosuppressive agents is a significant cause of patient morbidity and mortality $(1,2)$. In this regard, the induction and maintenance of allograft tolerance is the Holy Grail of transplantation. Over the past few decades, short-term allograft outcomes have remarkably improved under conventional immunosuppression. Thus, it could be argued that combining novel immunomodulatory protocols with short courses of immunosuppressive drugs that foster or facilitate tolerance is indeed justifiable.

The recent identification and characterization of regulatory $\mathrm{T}$ cells (T regs) has opened up exciting opportunities for tolerance induction, immunotherapy and immunomodulation in transplantation (3). $T$ regs possess great potential in the induction or promotion of tolerance while alleviating the need for immunosuppression and associated adverse effects (4). The discovery of T regs has also led to new insights into their role in solid organ and tissue transplant rejection and into mechanisms of tolerance breakdown in human diseases, including those resulting from allergic, autoimmune, or infectious causes (5). Thus, to the extent that active

(C) 2010 Elsevier Inc. All rights reserved

Corresponding Author: Xin Xiao Zheng BST W1557, 200 Lothrop Street, Pittsburgh PA, 15213 Telephone: 4126480177 Fax: 412634 6666.

Publisher's Disclaimer: This is a PDF file of an unedited manuscript that has been accepted for publication. As a service to our customers we are providing this early version of the manuscript. The manuscript will undergo copyediting, typesetting, and review of the resulting proof before it is published in its final citable form. Please note that during the production process errors may be discovered which could affect the content, and all legal disclaimers that apply to the journal pertain. 
immunologic mechanisms are required to induce transplantation tolerance, vigorous evaluation of the impact of conventional immunosuppressive drugs on $\mathrm{T}$ reg cell activation, proliferation and function is critical to develop new approaches and new paradigms for transplantation tolerance. Identifying and establishing a homeostatic balance between alloreactive $\mathrm{T}$ effectors (targeted through drug therapy) and T regs (mediating immunoregulation) could be the cornerstone to promote, induce or maintain tolerogenic mechanisms after transplantation.

\section{Tolerance in Transplantation}

Tolerance is a state where the immune system does not mount a pathologic response to specific antigens (eg graft antigens) while fully maintaining responses towards third party antigens (eg bacterial, viral or other nominal antigens) in the absence of extraneous ongoing immunosuppression. $\mathrm{T}$ cells, among other cell types, play an essential role in induction and maintenance of immune tolerance. In general, $\mathrm{CD} 4^{+} \mathrm{T}$ cells recognize peptide antigens presented by class II, and CD8 ${ }^{+} \mathrm{T}$ cells recognize peptide antigens presented by class I MHC molecules. Both $\mathrm{T}$ cell subsets collaborate in mediating immune responses.

Tolerance to self-antigens is not solely the result of total and permanent deletion of autoreactive $\mathrm{T}$ cells in the thymus. Indeed, in many circumstances immunoregulatory mechanisms participate in the maintenance of tolerance. In the case of transplantation across MHC barriers, $\mathrm{T}$ cells encounter allo-antigens bound to both self-MHC (indirect allo-recognition) and alloMHC molecules (direct allo-recognition). As a consequence, T-cell response to MHCmismatched allografts recruits a remarkably large number of T-cell clones (6). The magnitude of the number of $\mathrm{T}$ cells clones participating in the allograft response is exponentially larger than the $\mathrm{T}$ cell response mounted against nominal- or auto-Ags. In consideration of the remarkably large frequency of alloreactive T-cells, it seems intuitively correct that deletional mechanisms may play a key role in the induction phase of transplantation tolerance to MHCmismatched allografts. Although the precise mechanisms involved in the establishment and maintenance of allograft tolerance are still not fully understood, some mechanisms that have been implicated include ignorance, anergy, deletion, suppression and regulation (3). Transplantation tolerance is an active, highly regulated, multistep process.

High avidity or repeated TCR stimulation, however, can lead to T cell depletion through apoptosis both in the thymus and the periphery, referred to as central and peripheral deletion, respectively. Recent studies have shown that inhibition of $\mathrm{T}$ cell depletion leads to graft rejection, whereas strategies that promote $\mathrm{T}$ cell depletion may result in graft versus host disease (GVHD) (7).

$\mathrm{T}$ cell tolerance also includes non-deletional mechanisms. T cell competence requires a primary signal through the TCR plus a secondary signal through one or more co-stimulatory signals. If $\mathrm{T}$ cells encounter antigen stimulation alone, they will become functionally inactive. This mechanism is referred to as anergy. Many molecules, including CD28, inducible T cell costimulator (ICOS), CD40L and others can provide co-stimulation to T cells, and signals derived from those molecules are at least not completely redundant. Cumulative evidence indicates that these co-stimulatory signals are critical in promoting graft rejection or GVHD, as blockade of one or more of these co-stimulatory signals prevents or significantly delays these deleterious processes (8).

Over the past decade, $\mathrm{T}$ cell populations with regulatory functions have been linked to the prevention of autoimmunity. $T$ regs act in a dominant, trans-acting way to actively suppress immune activation, thereby functioning as critical mediators of self-tolerance. Further studies indicate that these $\mathrm{T}$ regs are also competent to suppress immune response to alloantigens, which opens up opportunities for new therapies in transplantation 


\section{Regulatory T Cells}

Regulatory $\mathrm{T}$ (Treg) cells are subsets of $\mathrm{T}$ cells involved in the maintenance of peripheral selftolerance by actively suppressing the activation and expansion of autoreactive $\mathrm{T}$ cells. Accumulating evidence indicates that more than one population of regulatory T-cells is engaged in the maintenance of peripheral tolerance $(9,10)$. These different regulatory populations function in different ways and some are naturally produced and other are locally induced as a result of immune responses (9). Several types of Treg cells have been characterized. The most prominent types are natural and inducible CD4+CD25+ Treg cells (11).

\section{Natural CD4+CD25+ Treg cells}

Compelling evidence indicates a key role for Treg cells in the maintenance of self-tolerance. These cells mediate dominant suppression of autoreactive $\mathrm{T}$ cells normally present in the periphery, as well as downregulating immune responses to foreign antigens (12). Among the several subpopulations of Treg cells identified to date, the naturally arising CD4+CD25+ Treg cells have emerged as being particularly critical for the maintenance of immunologic tolerance (13). CD4+CD25+ Treg cells arise in the thymus, represent 5\% to 10\% of CD4+ T cells in the periphery, and constitutively express the IL-2 receptor (IL-2R) $\square$ chain (CD25), cytotoxic T lymphocyte-associated antigen 4 (CTLA-4), and glucocorticoid-induced TNF receptor familyrelated gene (GITR). As detailed in this review, CD4+CD25+ Treg cells also express a transcriptional regulator, Foxp3, that acts as a master switch gene for their development and function. Consistent with their function in maintaining self-tolerance, depletion of CD4+CD25 + Treg cells precipitates autoimmunity against multiple tissues. CD4+CD25+ Treg cells are anergic and do not produce IL-2. When stimulated they suppress the proliferation and cytokine production of conventional CD4+CD25-T cells, as well as that of CD8+ T cells and established Thl and Th2 cells. CD4+CD25+ Treg cells produce TGF- $\beta$ and IL-10, 2 cytokines endowed with immunosuppressive functions that play critical functions in Treg cell biology. Suppression by CD4+CD25+ Treg cells can proceed by means of several mechanisms that might be differentially used depending on the microenvironment and the immunopathology being suppressed. In vitro, the immunosuppressive mechanism used by CD4+CD25+ Treg cells appears to be cell contact and CTLA-4 dependent but might proceed independently of IL-10 and TGF- $\tilde{\beta}$ In vitro-activated CD4+CD25+ Treg cells express granzyme A and display perforin-dependent cytotoxicity against autologous target cells, suggesting a function for direct killing (14). In contrast, suppression in several in vivo systems could be demonstrated not only to be CTLA-4 dependent but also IL-10 dependent, TGF- $\beta$ dependent, or both $(15,16)$. TGF$\beta$ might act by directly engaging TGF- $\beta$ receptor type II on target effector cells and might also be important for peripheral homeostasis of CD4+CD25+ Treg cells, indicating a pleiotropic function of this cytokine in supporting immunosuppression by Treg cells.

\section{CD4+CD25+ Treg Cell Development in the Thymus and Periphery}

Natural CD4+CD25+ Treg cells develop in the thymus (17). Neonatal thymectomy is associated with the development of autoimmunity secondary to CD4+CD25+ Treg cell deficiency (11). Mechanisms regulating CD4+CD25+ Treg cell development in the thymus remain unclear. One set of studies support a mechanism whereby CD4+CD25+ Treg cell development involves high-affinity interaction of their T-cell receptor (TCR) with peptide/ MHC ligands (18), especially those presented by thymic epithelial cells, at levels of avidity approaching those associated with clonal deletion. An alternative model suggests that the development of CD4+CD25+ Treg cells is induced by mechanisms other than recognition of self-agonist peptides but that $\mathrm{CD} 4+\mathrm{CD} 25+$ Treg cells are more resistant to agonist-induced 
clonal deletion, leading to the selective survival of CD4+CD25+ Treg cells at high-avidity TCR peptide-MHC Interactions (18).

In the periphery the majority of natural Treg cells constitutively express high levels of CD25 (CD25 $\left.5^{\text {high }}\right)$, but a significant minority express low levels of CD25 (CD25 low $)$. Both populations are immunosuppressive, and both express the transcription factor foxp3. It is thought that the $\mathrm{CD} 25^{\text {low }}$ population represents a population of natural Treg cells undergoing homeostatic proliferation, tissue Infiltration, or both. The maintenance of CD4+CD25+ Treg cells in the periphery is critically dependent on the action of two cytokines, IL-2 and TGF- $\beta$ (19). Although CD4+CD25+ Treg cells do not produce IL-2, they are dependent on this cytokine for development in the thymus and for homeostasis and activation of suppressor function in the periphery (see discussion later in this review). In studies on TGF- $\beta$ deficient mice, TGF- $\beta$ was found to be necessary to maintain foxp 3 expression, regulatory function, and pool size of peripheral CD4+CD25+ Treg cells but was not required for development of CD4+CD25+ Treg cells in the thymus (20).

\section{Natural versus Adaptive (or Inducible) CD4+CD25+ Treg Cells}

Several groups including our own have demonstrated an inability to obtain allograft tolerance in mice depleted of $\mathrm{CD} 4+\mathrm{CD} 25+$ natural Tregs prior to transplantation, indicating thymusderived Tregs play important roles in tolerance induction in these models. Other studies also suggested the existence of alloantigen-specific Tregs induced in the periphery. The natural and induced Tregs might be interrelated by an "infectious" tolerance mode first proposed by Dr. Herman Waldmann in 1993, where CD4+ T cells from tolerant mice disable naïve lymphocytes, which then become tolerant and, in turn, develop the capacity to antigenspecifically disable other naïve CD4+ cohorts (21). Recent studies showed that CD4+CD25+ Treg cells could be derived in vitro through treatment of peripheral CD4+CD25- T cells with anti-TCR and anti-CD28 antibodies in the presence of TGF- $\beta$. These so-called adaptive CD4 +CD25+ Treg cells express foxp3 and exhibit attributes typical of the natural CD4+CD25+ Treg cells, such as suppression of in vitro-induced antigen and mitogen-driven T-cell proliferation and down regulation of allergic lung inflammation induced by ovalbumin in transgenic mice expressing an ovalbumin peptide-specific TCR (22). Whereas the induction of murine adaptive CD4+CD25+ Treg cells in vitro by cross-linking of the TCR and CD28 stimulation is absolutely dependent on TGF- $\beta$, it has been reported that human adaptive CD4 $+\mathrm{C} 25+$ Treg cells can be induced in vitro in the absence of TGF- $\beta$, but this observation was not reproduced in independent studies.

The relationship between natural and adaptive CD4+CD25+ Treg cell populations is of significant interest. Do adaptive Treg cells derive from committed natural (thymically educated and committed). Treg cells that cycle between CD4+CD25+ and CD4+CD25- states in the periphery, or could they be derived de novo in an antigen-specific manner from non-Treg, naive T-cell population? At least two different in vivo experimental approaches indicate that adaptive Treg cells could be derived de novo from naive CD4- T cells in the periphery. Using thymectomized TCR-transgenic mice on a RAG knockout background, Apostolou and Von Boehmer demonstrated that CD4+CD25+ foxp3+ Treg cells could be derived de novo from peripheral CD4+ naive $\mathrm{T}$ cells through continuous infusion of a submitogenic concentration of an agonistic peptide. The second approach used RAG-deficient mice repopulated with monoclonal T- and B-cell populations that are otherwise devoid of natural CD4+CD25+ Treg cells (23). Whereas intraperitoneal immunization of these mice is associated with the induction of hyper-lgE and lung inflammation, oral antigen administration is associated with tolerance induction mediated by oral antigen-induced CD4+CD25+ Foxp3+ Treg cells. These results from both intro and in vivo experimental systems reveal a surprising plasticity of the CD4 $+\mathrm{CD} 25-$ populations to develop de novo into CD4+CD25+ Treg cells, with obvious 
implications for the treatment of human diseases with ex vivo-derived Treg populations or with immunotherapy, as is already used in allergic diseases.

\section{Foxp3, a Master Switch Gene for CD4+CD25+ Treg Cells}

The identification of the forkhead-type factor Foxp3 as a master switch gene for CD4+CD25 + Treg cells has provided a pivotal breakthrough in our understanding of CD4+CD25+ Treg cell development and function $(24,25)$. Foxp3 was originally identified as the gene product affected in a lethal X-linked recessive lymphoproliferative disease in mice and human subjects $(26,27)$. Loss-of-function mutations in the gene encoding Foxp3 underlie the

lymphoproliferative disease of the scurfy mouse. Male mice with Foxp3 deficiency die at about the third week of age because of unrelenting infiltrative lymphoproliferative disease that involves several organs. Foxp3-deficient mice also experience allergic dysregulation with striking hyper-lgE levels and eosinophilia in the absence of overt Th2 skewing (26). The striking hyper-lgE level is a distinguishing feature of CD4+CD25+ Treg cell deficiency in that it is shared by other mouse models lacking in CD4+CD25+ Treg cells. Importantly, the scurfy phenotype can be reproduced by targeted mutagenesis of Foxp3 and is rescued by a Foxp3 transgenic, which is consistent with the causative role for Foxp3 in disease pathogenesis.

FOXP3 is considered the most specific marker for Tregs and is crucial for suppressive activity of these cells (28). However, some non-regulatory T cells also transiently up regulate FOXP3 when activated. Methylation profiling of the FOXP3 promoter through bisulphite sequencing, or chromatin immunoprecipitation could be one method to evaluate stability of FOXP3 expression as well as suppressor ability (29). In one study (Janson PC, 2008), human CD4 +CD25 (hi) T regs displayed a demethylated FOXP3 promoter while CD4+CD25 (lo) T cells were partially methylated. When activated, these CD4+CD25 (lo) T cells showed transient FOXP3 expression but did not change their methylation pattern (30). This suggests that FOXP3 methylation is a good marker of FOXP3 stability and suppressor characteristics but also of the commitment of $\mathrm{T}$ regs in cultures.

FOXP3 mutations also underlie a homologous autoimmune lymphoproliferative disorder in human subjects, termed immune deregulation polyendocrinopathy enteropathy-X-linked (IPEX) syndrome and X-linked autoimmunity-allergic disregulation syndrome (XIAAD) (31). Male subjects with this syndrome present with neonatal autoimmune type 1 diabetes with islet cell destruction by infiltrating T cells. A more general predilection to autoimmunity is manifest, including polyendocrinopathy, autoimmune hemolytic anemia, and autoimmune enteropathy Another prominent feature of IPEX/XIAAO is severe allergic inflammation with eczema and food allergy. The IgE levels can be extremely increased and accompanied by intense peripheral eosinophilia and (unlike the case of the mouse models discussed below) evidence of overt Th2 skewing. The latter might reflect the evolution of the disease under the influence of ongoing immunosuppressive therapy. Many patients have persistent secretory diarrhea, which might be contributed to by both food allergy-induced eosinophilic gastroenteropathy and autoimmune inflammatory bowel disease-like enteropathy. Despite aggressive therapy, the outcome of IPEX/XIAAO is poor, with most patients dying in childhood. Bone marrow transplantation has been attempted in some patients and is potentially curative. In both mice and human subjects, female carriers are asymptomatic, which is consistent with $\mathrm{X}$-linked recessive inheritance. A more general question relates to the temporal requirement for Foxp3 expression to maintain natural CD4+CD25+ Treg cell phenotype in the periphery. Transgenic expression of Foxp3 in the thymus fails to rescue the lethal phenotype of Foxp3-deficient mice, which is consistent with a requirement for Foxp3 expression in the periphery. 
Emerging evidence associates decreased Foxp3 expression in Treg cells with various immune disorders. Utilizing a mouse model in which endogenous Foxp 3 gene expression is attenuated in Treg cells, Y Y Wan, et. al. showed that the decreased Foxp3 expression causes immune disease by subverting the suppressive function of Treg cells and converting Treg cells into effector cells; these findings are important for understanding the regulation of Treg cell function and the etiology of various human immune diseases (32).

\section{CD4+CD25+ Treg Cells versus other Treg Populations}

Several studies confirm the existence of multiple regulatory $\mathrm{T}$ cell populations with differential functions and produced naturally or upon immune induction. (9). Further characterization of the function and development of these cells will contribute to our understanding of the intrinsic and extrinsic mechanisms during an acquired process that maintain the peripheral tolerance.

\section{IL-10 T reg Cells (Tr1) and Th3 Cells}

The IL-10-secreting regulato ry T cells (IL-10 Treg cells) encompass a heterogeneous group of Treg cells that are induced in the periphery in response to antigenic stimulation and are characterized by the secretion of large amounts of IL-10 (33). A subgroup of IL-10 Treg cells are the Treg type 1 cells or $\operatorname{Tr} 1$ cells (34). These cells were originally identified in patients with severe combined immunodeficiency who have successfully undergone HLA-mismatched bone marrow transplantation. Tr1 cells secrete IL-10, IL-5, and IFN $\gamma$ but little IL- 2 and exert their suppressive function through an IL-10-dependent mechanism. Tr1 cells might play critical role in limiting pathologies associated with a high antigenic load, such as infections and severe inflammation. Other IL-10 Treg cells can be induced in vitro by antigenic or mitogenic stimulation in the presence of corticosteroids and vitamin D3 and secrete IL-10 but not other Thl or Th2 cytokines. These, too, are effective in suppressing experimental models of autoimmune disease, such as experimental allergic encephalomyelitis. Yet another Treg population, Th3, has been described that is preferentially generated after oral antigen administration and that secretes large amounts of TGF- $\beta$.

Currently, the relationship between the different Treg populations, including natural and adaptive CD4+CD $25+$ Treg cells, IL-10-secreting Treg cells, and TGF- $\beta$-secreting Th 3 cells, remains unclear. Unlike CD4+CD25+ Treg cells, IL-10-secreting Treg cells derived in vitro in the presence of dexamethasone and vitamin D3 express little or no foxp3. Nevertheless, it should be noted that corticosteroids have been described to induce foxp3, and there has yet to be a formal demonstration that IL-10-secreting Treg cells can be derived from lymphocytes of foxp3-deficient animals. It has been suggested that the IL-10- secreting Treg cells and CD4 $+\mathrm{CD} 25+$ Treg cells represent two separate and specialized populations, the first endowed with an anti-inflammatory capacity and infiltrates injured tissues to control inflammation and tissue destruction through the release of IL-10 and TGF- $\beta$, whereas the latter has a central homeostatic function to regulate $\mathrm{T}$-cell proliferation through direct cell-cell contact mechanisms (35). There have been very few studies that have compared these different populations head to head in the same disease models, and the results seem to indicate significant redundancy. The availability of mice whose Foxp3 expression is coupled with reporter fluorescent proteins and the application of genomic profiling approaches to the study of Treg cells offers to clarify the relationship between the different Treg populations.

In addition to its expression in CD4+ T cells, foxp3 is also expressed in a small population of CD8+ T cells. Foxp3+CD8+ Treg cells have been described in both human subjects and rodents and appear to share many of the phenotypic features with their CD4+CD25+ counterparts, including constitutive expression of CD2S, CTLA-4, and TGF- $\beta$. They have been implicated in the maintenance of transplant tolerance in rodent models of allograft rejection. 


\section{CD4-CD8- (double-negative, DN) T-cells}

In the peripheral lymphoid tissues of normal mice and humans, $1-5 \%$ of __ TCR+ T-cells are CD4-CD8- (double-negative, DN) T-cells (36),(37). The DN T-cells have been shown to possess the capacity to regulate auto- and alloimmune responses and induce immune tolerance (38-41). The heterogeneity of DN T-cells in the expression of surface markers suggests that several maturation/differentiation pathways may exist. In murine models several studies have demonstrated that DN TCR+ T-cells can be derived directly from CD8+ T-cells (42-45). Other studies suggest that DN _ TCR+ NKT cells arise extrathymically from bone marrow (46). More recently, Ford et al reported that DN T regulatory cells can develop outside the thymus, but not from mature CD8+ T-cell precursors (47). Moreover, recent study by Karin Fischer, et al, reported the isolation and characterization of human antigen-specific _ TCR+CD4-CD8 - DN regulatory $\mathrm{T}$ cells, the human counterpart of the DN T reg cells that have been studied in mice (48). They were able to isolate the DN T reg cells from PBMCs of adult healthy volunteers in sizable quantities (about 1\% of total CD3 T cells). Furthermore, the data of quantitative $\mathrm{T}$ cell receptor excision circles analysis indicate that the DN T reg cells are not recent thymic emigrants and in 3 of 7 healthy donors even had a longer proliferative history than either CD4 or CD8 T cells (48).

In our recent study, we uncovered a new differentiation pathway of DN regulatory $\mathrm{T}$ cells. Our evidence shows that a subset of proliferating CD4+ T-cells converted to DN regulatory cells after 4-5 rounds of either antigen triggered or homeostatic proliferation in vitro and in vivo (49). The CD4+ T cell converted DN regulatory $\mathrm{T}$ cells are very potent in inhibiting allo and autoimmune responses in a antigen specific manner; and perforin plays a role in DN T cells mediated cell killing and suppression. This pathway represents a new intrinsic homeostatic mechanism that regulates the magnitude of immune responses to alloantigen both in vitro and in vivo and will permit the development of novel, cell-based, therapeutic approaches for the prevention of allograft rejection and for the treatment of autoimmune diseases.

\section{Indoleamine 2,3-dioxygenase (IDO) and Tregs}

Indoleamine 2,3-dioxygenase (IDO) is the rate-limiting enzyme in the degradation of the essential amino acid tryptophan via the kynurenine pathway. IDO is widely distributed in mammals and is induced in various cell types, predominantly by the Th1-type cytokine interferon- $\gamma($ IFN- $\gamma)(50)$. For many years, IDO has been known as an innate defence mechanism limiting growth of viruses, bacteria, or malignant cells by withdrawing tryptophan from the local microenvironment $(51,52)$. In more recent studies, it has been proposed that activation of IDO is also critically involved in regulating immune responses (53), establishing immune tolerance in pregnant mice upon their fetuses (54), or inducing T-cell unresponsiveness (55). Proliferation of alloreactive T-cells is thereby arrested within the G1 phase via local tryptophan deprivation and the accumulation of pro-apoptotic catabolites such as kynurenine and quinolinic acid (56). In addition, IDO has been found to serve as a potent downstream suppressor mechanism used by T-regulatory cells (Treg) (57).

Treg are known to have a high constitutive expression of the cytotoxic T-lymphocyte antigen-4 (CTLA4), and several studies have indicated that CTLA4 activity is essential for Treg to mediate their immunomodulatory and pro-tolerogenic function (58).

Recently a report by Fallarino et al. provided evidence that $\mathrm{CD} 4{ }^{+} \mathrm{CD} 25^{+}$Treg can condition DCs to release IFN- $\gamma$ and to induce IDO through a CTLA4-dependent mechanism hence facilitating immunosuppressive properties (57). The authors propose a distinct pathway by which CTLA4-expressing Treg might affect the properties of IDO ${ }^{+}$DCs through B7 signalling. Also, a cloned $\mathrm{CD}^{+} \mathrm{CD} 25^{+} \mathrm{T}$-cell line, expressing surface CTLA4, induced IDO expression within $\mathrm{B} 220^{+}$and $\mathrm{CD} 8 \alpha^{+}$DCs. Regulatory functions of these T-cells were found to be strictly 
dependent on their ability to induce IDO expression within DCs (59). Taken together these studies provide a novel emerging concept for the immune privilege induced by Treg in a way that Treg and $\mathrm{IDO}^{+}$regulatory or tolerogenic DCs might vitally interact to form an immunomodulatory network, capable of suppressing local T-cell responses and promoting systemic tolerance.

\section{Regulatory T Cells in Solid Organ and Composite Tissue Allotransplantation}

In contrast to the transplantation of haematopoietic stem cells, where mutual tolerance between recipient and graft usually ensues after a short period of time, lifelong immunosuppression is usually necessary in solid organ transplantation to allow survival of the transplanted organ. Despite treatment with immunosuppressive drugs, chronic graft failure still represents a major problem. Creating a state of mixed haematopoietic chimaerism has been shown to be able to induce deletional tolerance for organ grafts $(60,61)$. However, this approach is limited in its feasibility for obvious reasons.

Recently, there is an emerging consensus that immunoregulation plays a critical role in dominant tolerance in organ transplantation (62). Multiple reports have established that activation of CD4+CD25+ Tregs constitutes an essential element of the immunoregulatory pathways that create peripheral allograft tolerance (63). In the absence of Tregs, grafts are rejected in situations where they would otherwise be tolerated. Recently emerged importance of regulatory $\mathrm{T}$ cell (Treg cell) in allograft tolerance has lead to the concept that the balance between allo-aggressive cytopathic $\mathrm{T}$ cell population and allospecific Treg cell population is crucial to prevent allogeneic graft rejection and go on to graft tolerance (64-68). Enhanced allospecific Treg cell function has been reported to be necessary to maintain the allograft tolerance in several successful experimental tolerogenic regimens, and the tools to effectively inducing or expanding allospecific Treg cell are under extensive exploration. One of the most actively investigated tolerance induction regimen regarding regulatory $\mathrm{T}$ cell is donor specific transfusion (DST) and anti-CD154 monoclonal antibody treatment. Along with depletion or anergy induction of alloreactive T cells, eliciting Treg cell function has been reported (6870). The observation of graft loss after deleting the Treg cells in animals induced tolerance with this regimen (70) and prolonged skin graft survival in T cell-deficient mice reconstituted with naïve effector T cells (Teff cells) along with Treg cells treated with DST and anti-CD154 (CD40L) one month before, but not with treated Teff cells (71), suggest that enhancing regulatory $\mathrm{T}$ cell function would be the main mechanism of tolerance induction in this regimen.

The clinical importance of Tregs was shown by Yoshizawa et al who demonstrated potent CD4 +CD25+ Tregs in liver transplant recipients who could be weaned off immunosuppression (72). Other reports have also associated long-term survival of human allografts with the presence of Tregs in the peripheral blood. Bringing these findings closer to a clinically applicable immunotherapy with Tregs, Karim et al observed that bystander regulation leading to cardiac allograft acceptance can be achieved by Tregs that have been induced by exposure to an irrelevant protein antigen (73). In CTA, a study examining skin biopsies six years after clinical hand transplantation reported the presence of intragraft $\mathrm{T}$ regs (manifesting as a lymphocytic infiltrate) with positive FOXP3 mRNA expression (74). Moreover, suppressive cytokine profile was detected in the transplanted skin without evidence of perforin mRNA expression supporting a putative role of FOXP3+ T regs in long-term graft survival or tolerance. The cytokine mRNA profile demonstrated increased levels of TGF beta and IL-10 mRNA expression in fresh biopsies, with no or very low levels of TNF alpha, or IFN gamma. Taken together these studies not only indicate a pivotal role for Tregs in solid organ transplantation and CTA tolerance, but also pave the way for future clinical studies in this area. 
As lymphocytes capable of prolonging graft survival upon adoptive transfer into naïve graft recipients have been detected in rejecting hosts, suggests that activation of $\mathrm{T}$ reg cells is an integral component of the allograft response. More recent studies demonstrated in a primate kidney transplant model that the degree of FOXP3 expression showed significant positive correlation with the Banff grade of rejection as well as with surrogate markers like creatinine levels (74). Similar findings were observed in clinical cardiac transplant patients where FOXP3 mRNA expression levels correlated with the histologic grade of rejection in endomyocardial biopsies (75). These observations suggest that FOXP3+ T cells do not prevent acute rejection, but rather are a response to anti-donor effector T-cell activity. Most studies in hand transplant patients assumed that the presence of Foxp3+Tregs in rejected tissues is a physiological reaction to an inflammatory response and to downregulate the effector T cells. An elegant study by Muthukumar reported the FOXP3 mRNA levels of urine samples of renal transplantation patients were inversely correlated with serum creatinine levels measured at the time of biopsy in the acute-rejection group but not in the group with chronic allograft nephropathy or the group with normal biopsy results. Moreover, the measurement of FOXP3 mRNA in urine also predict the outcome of acute rejection of renal transplants.(76) These results indicated that activation of $\mathrm{T}$ reg cells is an integral component of the allograft response. In the absence of effective therapy, however, the more rapid expansion of alloagressive T-cells overcomes the protective effects of Treg cells. We hypothesize that the allograft outcome, rejection or tolerance, often depends on the balance between cytopathic and regulatory T cells. $(64-66,68,77)$ Due to the large numbers of alloreactive T cells responding against allogeneic MHC molecules $(6,78)$ the induction of peripheral tolerance requires trimming of the pool size of alloreactive $\mathrm{T}$ cells in order to allow regulatory $\mathrm{T}$ cells to exert their suppressive effects. $(68,79)$.

Although both deletion and regulation play important roles in allograft tolerance, the quantitative details for each mechanism differs from model to model. As in both allograft tolerance models in which allograft tolerance is induced by either DST plus MR1 or combined RPM plus lytic IL-2/Fc and mutIL-15/Fc, the deletion of CD4+CD25+ Treg cells results in acute allograft rejection, indicating that regulatory network is critical for the allograft tolerance induction in these models. In contrast, the deletion of CD4+CD25+ Treg cells does not interfere the establishment of stable chimera and skin allograft tolerance in the recipients treated with BMT, RPM and costimulation blockade, suggesting that regulatory network (at least CD4 $+\mathrm{CD} 25+$ dependent immunoregulation) is not required for the tolerance induction in this model. On the other hand, while the CD4+CD25+ Tregs from DST plus MR1 treated hosts demonstrate more powerful alloantigen specific suppressive function per cell to cell basis than that obtained from naïve mice, the capacity of CD4+CD25- effector T cells to trigger skin allograft rejection per cell to cell bases is identical to that from naïve mice, suggesting that the deletion is not nearly complete in this model. In a mixed chimerism and skin allograft tolerance model induced by BMT, RPM and costimulation blockade, although we can clearly demonstrate the present of regulatory network, the adoptive transfer of CD4+CD25- T cells from tolerance mice into syngeneic $\mathrm{Rag}-/-$ mice does not trigger skin allograft rejection, suggesting the deletion of alloaggressive $\mathrm{T}$ cells is more profound in this model.

In accordance to the pool size model of allograft tolerance, the allograft outcome, rejection or tolerance, often depends on the balance between cytopathic and regulatory T cells. Although both deletion and regulation play important roles in allograft tolerance, our recent studies showed that the quantitative details for each mechanism differs from model to model. Therefore, we hypothesize that there is a delicate balance between deletion and regulation in allograft tolerance. As in a model of allograft tolerance in which the deletional mechanism play a dominant role, e.g. tolerance produced via creation of mixed chimeras, the regulatory mechanisms, albeit sometimes present, are far less important. While in a model in which the regulation mechanism plays critical role, e.g. DST plus MR1 induced allograft tolerance, deletional mechanisms lowers the threshold for effective $\mathrm{T}$ reg action (Figure 4). 
Therefore, the development of clinical monitoring assays to quantitatively determine the balance of alloagressive versus regulatory immune responses will provide meaningful information to adjust the treatment in transplant recipients.

\section{Effect of Immunosuppressive Drugs on T regs}

Newer drugs such as cyclosporine (CsA), tacrolimus, mycophenolate mofetil (MMF), and rapamycin (RPM) are very effective in preventing allograft rejection and are major contributors to improved short-term outcome. In general, these agents are preventing all types of immune responses, both destructive ones and potentially protective ones. However, since the remarkable improvement of short-term allograft outcome by administering conventional immunosuppressive drugs, it would be ethically required in the clinical setting of organ transplantation to combine new tolerant protocol with short course of immunosuppressive drugs. Therefore, vigorous evaluation of the impact of conventional immunosuppressive drugs on Tregs activation, proliferation and function is critical to develop new approaches and new paradigms for transplantation tolerance.

Calcineurin inhibitors, such as cyclasporin A and (tacrolimus, inhibit immune responses and prevent rejection by blocking the transcription of the gene, encoding for IL-2. Since IL-2 was found to be necessary for long-term allograft survival as well as for the survival and function of Tregs, there is a valid concern about the potential negative impact of calcineurin inhibitors on Tregs. In fact, recent studies have shown that cyciosporin A may inhibit the development of Tregs and may also interfere with the suppressive activity of Tregs. While we do not prefer calcineurin inhibitor based protocols as the best means to gain peripheral type transplant tolerance, our recent study does suggest a interim means by which calcineurin inhibitors may be used in conjunction with IL-2/Fc in attempt to create tolerance (80).

The key for the success of this protocol in rodents may lie in the fact that CsA was given in a 14-day short-term course, not as a maintenance therapy. During this 2-week period, the CsA plus IL-2/Fc regimen allows exaggerated activation-induced cell death (AICD) of effector T cells and enhances allospecific immunoregulatory function of Tregs that are pre-requisite for tolerance induction. After the short course of CsA and IL-2/Fc treatment in the tolerance induction phase, secession of CsA treatment would permit the "infectious" tolerance to take place and recruit new army of induced Tregs to achieve dominant tolerance (80). Our work may enlarge the pool of rational therapeutic regimens at hand in the attempts of the transplant community to achieve tolerance. Interestingly, rapamycin selectively facilitates expansion of Tregs and preserves the suppressive activity of human Tregs in vitro, as does its derivative everolimus. Mycophenolate mofetil is another immunosuppressive drug commonly used in clinical transplantation. In an experimental study in mice, this drug has been shown to promote an increase in the frequency of Tregs and to induce transplantation tolerance when combined with $1 \square 2 \tilde{5}$ dihydroxyvitamin D3.

Monoclonal antibodies specific for $\mathrm{CD} 3$ have been used as therapeutic agents in autoimmune diseases, GVHD and organ transplantation. The frequency of Tregs was actually increased correlating with the protection from diabetes in clinical studies or in experimental autoimmune encephalomyelitis after anti-CD3 treatment. Costimulation through CD28, ICOS, CTLA-4, CD40L and others plays a critical role in T cell Immunity and tolerance, and manipulation of one or more of those signals has a great potential to be used as a therapeutic approach in transplantation. As discussed earlier, these costimulatory molecules also have a profound effect on the development, survival and/or function of Tregs, and, thus, the complex of these molecules on effector $\mathrm{T}$ cells and Tregs is still elusive and remains to be further investigated. 


\section{Promise and Pitfalls for Use of $\mathrm{T}$ regs in Transplantation}

Despite the abundance of evidence that Treg can be used therapeutically in models of autoimmunity, BMT and solid organ transplantation, it has been difficult to show that Treg can be used to induce tolerance. Notably, there has been no demonstration of allospecific tolerance induction by infusion of Treg in an otherwise unmanipulated, fully MHC incompatible host. There are many reasons that might explain this negative outcome. First, a significant barrier to Treg mediated tolerance is the high precursor frequency of alloreactive pathogenic T cells, which is estimated to be as high as 5-10\% in fully allogeneic combinations. This precursor frequency is at least several orders of magnitude greater than that of nominal antigen-specific T-cell responses. Thus, if tolerance or rejection is in part determined by the balance between Treg and Teff (Treg/Teff ratio), far more Treg may be needed to induce tolerance to alloantigens than autoantigens. This may explain why Treg therapy is highly effective in nonmanipulated models of autoimmunity, but has been relatively unsuccessful in nonmanipulated major mismatch transplantation models. Interestingly, spontaneous acceptance of cardiac allografts across a single MHC II disparity was shown to be mediated by Treg, consistent with the speculation that, under conditions of diminished precursor frequency, the Treg response can prevail over the Teff response for some graft types. The importance of the balance between Treg and Teff may explain why efficacy of therapeutic Treg in transplantation has been limited primarily to co adoptive transfer models into irradiated or genetically Iymphopenic hosts, wherein the number of Teff is dramatically reduced. Thus, it is likely that depletion of alloreactive Teff responses via apoptosis or anergy will be important for the induction of tolerance using Treg.

A second issue unique to transplantation is the presence of the direct and indirect pathways of antigen presentation. Treg with direct specificity as well as indirect specificity have been shown to prolong allograft survival in murine models. It will be important to determine whether Treg with direct and indirect specificity will have synergistic effects, and whether they will have differential effects on acute and chronic rejection. If the effect of Treg in vivo is primarily via modulation of DC, Treg with indirect specificity are not likely to significantly affect donorderived DC, while Treg with direct specificity may not be able to regulate self-DC presenting donor peptides. Treg with direct specificity are likely to have a much higher precursor frequency than Treg with indirect specificity, similar to the Teff cells. Thus, isolation and expansion of Treg with indirect specificity is likely to be challenging. However, techniques for antigen-specific expansion of Treg have been reported, suggesting that producing sufficient numbers of allospecific Treg for potential therapy will be feasible.

A third issue is that inflammatory signals associated with surgical trauma as well as ischemiareperfusion injury may alter the inhibitory activity of Treg while augmenting the T effector response. For example, TLR engagement on DC has been shown to prevent suppression by Treg in an IL-6-dependent manner. The engagement of TLR and systemic release of IL-6 has been well documented after transplantation. IL- 6 may also prevent the conversion of CD4 +Foxp3- cells into CD4+ Foxp3+ Treg. Recently, Chen et al showed that TLR engagement prevents anti-CDl54-mediated tolerance in a murine heart transplant model. This result correlated with decreased recruitment of Treg to the graft. In other studies, the presence of IL-6 and TGF- $\beta$ shift $\mathrm{T}$ cell differentiation away from Treg toward IL-17-producing, pathogenic Th17 cells. Reciprocally, Treg appear to be able to mitigate innate immune responses, and therapeutic Treg administration has been shown to decrease innate immune injury and promote engraftment in an islet transplant model. These results suggest that minimization of ischemiarepertusion injury will be a key element of tolerance induction strategies. Further studies will also need to address issues that are important in the clinical setting, but are generally not modeled in rodent studies for example; the effectiveness of Treg on memory $\mathrm{T}$ cells has not been clearly delineated. The effect of Treg on the generation of alloantibodies is also largely 
unknown. Finally, application of Treg therapy to the clinical setting will require a better understanding of Treg in clinical transplantation where patients are receiving chronic immunosuppressive therapy. Recent studies have suggested a potential role for Treg in regulating allograft rejection. Suthanthiran's group reported that levels of urinary mRNA for foxp 3 were correlated with the reversal of acute rejection in renal transplant patients receiving conventional immunosuppressive therapy. Similarly, the absolute numbers of Treg were increased in the allograft tissue of patients undergoing acute graft rejection, with higher numbers in those patients that responded effectively to anti-rejection therapy (Belingheri $\mathrm{M}$, Vincenti F, Gross and Bluestone JA, unpublished observations). The elucidation of drugs that inhibit Teff function while maintaining or even enhancing Treg activity will be critical to Treg therapy. Rapamycin has been shown to promote expansion of human Treg in vitro, and to preserve Treg numbers in mouse models as well as in renal transplant patients. On the other hand, calcineurin inhibitors appear to reduce the numbers of Treg in these patients.

Mycophenolate mofetil in conjunction with vitamin D3 appears to favor induction of Treg in mouse models, but its effect in humans is not well established. Another commonly used agent, antithymocyte globulin, has recently been shown to preferentially expand Treg in vitro, optimally at concentrations generally lower than observed in patients. However, a recent shortterm study in humans showed that Treg were efficiently depleted by both antithymocyte globulin and aletuzumab, which correlates with the lack of convincing data that these agents promote tolerance. Nevertheless, it is possible that these agents will be useful for Iymphodepletion before Treg therapy, in order to increase the Treg/Teff ratio. The effect of Abatacept (CTLA4Ig and the related drug Belatacept, on Treg is not yet clear. There is a concern that Treg may be depleted by B7 blockade, given the demonstration that CD28-B7 interactions are critical to the generation and survival of Treg. However, preliminary evidence suggests that combined Belatacept and anti-IL-2 receptor antibody treatment does not chronically deplete Treg after renal transplantation, likely because the blockade is nonsaturating.

\section{Regulatory T cells: Future Hopes and Potential Strategies}

Over the past decade, $\mathrm{T}$ regs have been widely touted as the key mediators of induction and/ or maintenance of peripheral self-tolerance. The current focus is to target specific immune responses in autoimmunity or transplantation with $\mathrm{T}$ regs. However, this is complicated by the fact that induction and regulation of immunity and tolerance to self or to grafts is greatly influenced by cytokines and other soluble mediators. The key to success is to first to identify and understand the key functional differences between various $\mathrm{T}$ reg populations and their representative markers. The secondary step will be to achieve the critical balance between immune enhancement and tolerance by determining how directed manipulation of specific $\mathrm{T}$ reg populations could tip the balance towards tolerance versus immunity. One of the chief obstacles to these goals and their clinical translation has been the differences in experimental results as obtained in mice compared to humans. One strategy to overcome this hurdle is to investigate ex-vivo means to generate discrete populations of $\mathrm{T}$ regs with defined specificity, function and downstream effects and then utilize these cells for clinical application via means like adoptive therapy. The other more difficult but potentially attractive approach is manipulating regulatory populations in vivo in patients suffering from autoimmune disease or graft rejection. Only time will tell how emerging insights will influence the scientific trends and approaches in the field of T reg immunology as applied to transplantation biology.

\section{Acknowledgments}

Supported in part by: U.S. National Institute of Health, U.S. Armed Forces Institute of Regenerative Medicine, Plastic Surgery Educational Foundation, and Juvenile Diabetes Research Foundation. 


\section{References}

1. Nankivell BJ, Borrows RJ, Fung CL, O'Connell PJ, Allen RD, Chapman JR. The natural history of chronic allograft nephropathy. N Engl J Med 2003;349:2326-2333. [PubMed: 14668458]

2. Wiesner R, Rabkin J, Klintmalm G, et al. A randomized double-blind comparative study of mycophenolate mofetil and azathioprine in combination with cyclosporine and corticosteroids in primary liver transplant recipients. Liver Transpl 2001;7:442-450. [PubMed: 11349266]

3. Wood K, Sakaguchi S. Regulator T cells in Transplantation Tolerance. Nat Rev Immunol 2003;3:199, 210. [PubMed: 12658268]

4. Joffre O, Santolaria T, Calise D, et al. Prevention of acute and chronic allograft rejection with CD4 +CD25+Foxp3+ regulatory T lymphocytes. Nat Med 2008;14:88-92. [PubMed: 18066074]

5. Vignali DACL, Workman CJ. How regulatory T cells work. Nat Rev Immunol 2008;8:523-532. [PubMed: 18566595]

6. Suchin EJ, Langmuir PB, Palmer E, Sayegh MH, Wells AD, Turka LA. Quantifying the frequency of alloreactive T cells in vivo: new answers to an old question. J Immunol 2001;166:973-981. [PubMed: 11145675]

7. Kolb HJ. Graft-versus-leukemia effects of transplantation and donor lymphocytes. Blood 2008;112:4371-4383. [PubMed: 19029455]

8. Ford ML, Larsen CP. Translating costimulation blockade to the clinic: lessons learned from three pathways. Immunol Rev 2009;229:294-306. [PubMed: 19426229]

9. Jiang H, Chess L. Regulation of immune responses by T cells. N Engl J Med 2006;354:1166-1176. [PubMed: 16540617]

10. Bluestone JA, Mackay CR, O'Shea JJ, Stockinger B. The functional plasticity of T cell subsets. Nat Rev Immunol 2009;9:811-816. [PubMed: 19809471]

11. Sakaguchi S. Regulatory T cells: key controllers of immunologic self-tolerance. Cell 2000;101:455458. [PubMed: 10850488]

12. Miyara M, Sakaguchi S. Natural regulatory T cells: mechanisms of suppression. Trends Mol Med 2007;13:108-116. [PubMed: 17257897]

13. Shevach EM, DiPaolo RA, Andersson J, Zhao DM, Stephens GL, Thornton AM. The lifestyle of naturally occurring CD4+ CD25+ Foxp3+ regulatory T cells. Immunol Rev 2006;212:60-73. [PubMed: 16903906]

14. Grossman WJVJ, Barchet W, Colonna M, Atkinson JP, Ley TJ. Human T regulatory cells can use the perforin pathway to cause autologous target cell death. Immunity 2004;21:589-601. [PubMed: 15485635]

15. Hara M, Kingsley CI, Niimi M, et al. IL-10 is required for regulatory T cells to mediate tolerance to alloantigens in vivo. J Immunol 2001;166:3789-3796. [PubMed: 11238621]

16. Belghith M, Bluestone JA, Barriot S, Megret J, Bach JF, Chatenoud L. TGF-beta-dependent mechanisms mediate restoration of self-tolerance induced by antibodies to $\mathrm{CD} 3$ in overt autoimmune diabetes. Nat Med 2003;9:1202-1208. [PubMed: 12937416]

17. Nomura T, Sakaguchi S. Foxp3 and Aire in thymus-generated Treg cells: a link in self-tolerance. Nat Immunol 2007;8:333-334. [PubMed: 17375092]

18. Fontenot JD, Rudensky AY. A well adapted regulatory contrivance: regulatory T cell development and the forkhead family transcription factor Foxp3. Nat Immunol 2005;6:331-337. [PubMed: 15785758]

19. Shevach EM. Mechanisms of foxp3+ T regulatory cell-mediated suppression. Immunity 2009;30:636-645. [PubMed: 19464986]

20. Marie JC, Letterio JJ, Gavin M, Rudensky AY. TGF-beta1 maintains suppressor function and Foxp3 expression in CD4+CD25+ regulatory T cells. J Exp Med 2005;201:1061-1067. [PubMed: 15809351]

21. Qin S, Cobbold SP, Pope H, et al. "Infectious" transplantation tolerance. Science 1993;259:974-977. [PubMed: 8094901]

22. D'Alessio FR, Tsushima K, Aggarwal NR, et al. CD4+CD25+Foxp3+ Tregs resolve experimental lung injury in mice and are present in humans with acute lung injury. J Clin Invest 2009;119:28982913. [PubMed: 19770521] 
23. Apostolou I, von Boehmer H. In vivo instruction of suppressor commitment in naive T cells. J Exp Med 2004;199:1401-1408. [PubMed: 15148338]

24. Ramsdell F. Foxp3 and natural regulatory T cells: key to a cell lineage? Immunity 2003;19:165-168. [PubMed: 12932350]

25. Huehn J, Polansky JK, Hamann A. Epigenetic control of FOXP3 expression: the key to a stable regulatory T-cell lineage? Nat Rev Immunol 2009;9:83-89. [PubMed: 19114986]

26. Brunkow ME, Jeffery EW, Hjerrild KA, et al. Disruption of a new forkhead/winged-helix protein, scurfin, results in the fatal lymphoproliferative disorder of the scurfy mouse. Nat Genet 2001;27:6873. [PubMed: 11138001]

27. Bennett CL, Christie J, Ramsdell F, et al. The immune dysregulation, polyendocrinopathy, enteropathy, X-linked syndrome (IPEX) is caused by mutations of FOXP3. Nat Genet 2001;27:2021. [PubMed: 11137993]

28. Onishi Y, Fehervari Z, Yamaguchi T, Sakaguchi S. Foxp3+ natural regulatory T cells preferentially form aggregates on dendritic cells in vitro and actively inhibit their maturation. Proc Natl Acad Sci U S A 2008;105:10113-10118. [PubMed: 18635688]

29. Floess S, Freyer J, Siewert C, et al. Epigenetic control of the foxp3 locus in regulatory T cells. PLoS Biol 2007;5:e38. [PubMed: 17298177]

30. Janson PC, Winerdal ME, Marits P, Thorn M, Ohlsson R, Winqvist O. FOXP3 promoter demethylation reveals the committed Treg population in humans. PLoS One 2008;3:e1612. [PubMed: 18286169]

31. Bennett CL, Brunkow ME, Ramsdell F, et al. A rare polyadenylation signal mutation of the FOXP3 gene (AAUAAA-->AAUGAA) leads to the IPEX syndrome. Immunogenetics 2001;53:435-439. [PubMed: 11685453]

32. Wan YY, Flavell RA. Regulatory T-cell functions are subverted and converted owing to attenuated Foxp3 expression. Nature 2007;445:766-770. [PubMed: 17220876]

33. Groux H, O'Garra A, Bigler M, et al. A CD4+ T-cell subset inhibits antigen-specific T-cell responses and prevents colitis. Nature 1997;389:737-742. [PubMed: 9338786]

34. Groux H. Type 1 T-regulatory cells: their role in the control of immune responses. Transplantation 2003;75:8S-12S. [PubMed: 12819483]

35. Roncarolo MG, Bacchetta R, Bordignon C, Narula S, Levings MK. Type 1 T regulatory cells. Immunol Rev 2001;182:68-79. [PubMed: 11722624]

36. Zhang ZX, Young K, Zhang L. CD3+CD4-CD8- alphabeta-TCR+ T cell as immune regulatory cell. J Mol Med 2001;79:419-427. [PubMed: 11511972]

37. Zhang ZX, Yang L, Young KJ, DuTemple B, Zhang L. Identification of a previously unknown antigen-specific regulatory $\mathrm{T}$ cell and its mechanism of suppression. Nat Med 2000;6:782-789. [PubMed: 10888927]

38. Born W, Cady C, Jones-Carson J, Mukasa A, Lahn M, O'Brien R. Immunoregulatory functions of gamma delta T cells. Adv Immunol 1999;71:77-144. [PubMed: 9917911]

39. Drobyski WR, Vodanovic-Jankovic S, Klein J. Adoptively transferred gamma delta T cells indirectly regulate murine graft-versus-host reactivity following donor leukocyte infusion therapy in mice. J Immunol 2000;165:1634-1640. [PubMed: 10903774]

40. Strober S, Cheng L, Zeng D, et al. Double negative (CD4-CD8- alpha beta+) T cells which promote tolerance induction and regulate autoimmunity. Immunol Rev 1996;149:217-230. [PubMed: 9005216]

41. Yang L, DuTemple B, Gorczynski RM, Levy G, Zhang L. Evidence for epitope spreading and active suppression in skin graft tolerance after donor-specific transfusion. Transplantation 1999;67:14041410. [PubMed: 10385077]

42. Takahama Y, Kosugi A, Singer A. Phenotype, ontogeny, and repertoire of CD4-CD8- T cell receptor alpha beta + thymocytes. Variable influence of self-antigens on $\mathrm{T}$ cell receptor $\mathrm{V}$ beta usage. $\mathrm{J}$ Immunol 1991;146:1134-1141. [PubMed: 1825105]

43. Landolfi MM, Van Houten N, Russell JQ, Scollay R, Parnes JR, Budd RC. CD2-CD4-CD8-lymph node $\mathrm{T}$ lymphocytes in MRL lpr/lpr mice are derived from a CD2+CD4+CD8+ thymic precursor. $\mathrm{J}$ Immunol 1993;151:1086-1096. [PubMed: 7687614] 
44. Balomenos D, Rumold R, Theofilopoulos AN. The proliferative in vivo activities of lpr doublenegative $\mathrm{T}$ cells and the primary role of p59fyn in their activation and expansion. J Immunol 1997;159:2265-2273. [PubMed: 9278315]

45. Bix M, Coles M, Raulet D. Positive selection of V beta 8+ CD4-8- thymocytes by class I molecules expressed by hematopoietic cells. J Exp Med 1993;178:901-908. [PubMed: 8350060]

46. Dejbakhsh-Jones S, Okazaki H, Strober S. Similar rates of production of T and B lymphocytes in the bone marrow. J Exp Med 1995;181:2201-2211. [PubMed: 7760006]

47. Ford MS, Zhang ZX, Chen W, Zhang L. Double-Negative T Regulatory Cells Can Develop Outside the Thymus and Do Not Mature from CD8+ T Cell Precursors. J Immunol 2006;177:2803-2809. [PubMed: 16920915]

48. Fischer K, Voelkl S, Heymann J, et al. Isolation and characterization of human antigen-specific TCR alpha beta+ CD4(-)CD8- double-negative regulatory T cells. Blood 2005;105:2828-2835. [PubMed: 15572590]

49. Zhang D, Yang W, Degauque N, Tian Y, Mikita A, Zheng XX. New differentiation pathway for double-negative regulatory $\mathrm{T}$ cells that regulates the magnitude of immune responses. Blood 2007;109:4071-4079. [PubMed: 17197428]

50. Byrne GI, Lehmann LK, Kirschbaum JG, Borden EC, Lee CM, Brown RR. Induction of tryptophan degradation in vitro and in vivo: a gamma-interferon-stimulated activity. J Interferon Res 1986;6:389-396. [PubMed: 3095441]

51. Pfefferkorn ER. Interferon gamma blocks the growth of Toxoplasma gondii in human fibroblasts by inducing the host cells to degrade tryptophan. Proc Natl Acad Sci U S A 1984;81:908-912. [PubMed: 6422465]

52. Ozaki Y, Edelstein MP, Duch DS. Induction of indoleamine 2,3-dioxygenase: a mechanism of the antitumor activity of interferon gamma. Proc Natl Acad Sci U S A 1988;85:1242-1246. [PubMed: 3124115]

53. Hwu P, Du MX, Lapointe R, Do M, Taylor MW, Young HA. Indoleamine 2,3-dioxygenase production by human dendritic cells results in the inhibition of T cell proliferation. J Immunol 2000;164:35963599. [PubMed: 10725715]

54. Munn DH, Zhou M, Attwood JT, et al. Prevention of allogeneic fetal rejection by tryptophan catabolism. Science 1998;281:1191-1193. [PubMed: 9712583]

55. Mellor AL, Munn D, Chandler P, et al. Tryptophan catabolism and T cell responses. Adv Exp Med Biol 2003;527:27-35. [PubMed: 15206713]

56. Munn DH, Shafizadeh E, Attwood JT, Bondarev I, Pashine A, Mellor AL. Inhibition of T cell proliferation by macrophage tryptophan catabolism. J Exp Med 1999;189:1363-1372. [PubMed: 10224276]

57. Fallarino F, Grohmann U, Hwang KW, et al. Modulation of tryptophan catabolism by regulatory $\mathrm{T}$ cells. Nat Immunol 2003;4:1206-1212. [PubMed: 14578884]

58. Sansom DM, Walker LS. The role of CD28 and cytotoxic T-lymphocyte antigen-4 (CTLA-4) in regulatory T-cell biology. Immunol Rev 2006;212:131-148. [PubMed: 16903911]

59. Baban B, Hansen AM, Chandler PR, et al. A minor population of splenic dendritic cells expressing CD19 mediates IDO-dependent T cell suppression via type I IFN signaling following B7 ligation. Int Immunol 2005;17:909-919. [PubMed: 15967784]

60. Ildtad ST, Sachs DH. Reconstitution with syngeneic plus allogeneic or zenogeneic bone marrow leads to specific acceptance of allografts or xenografts. Nature 1984;307:168-171. [PubMed: 6361574]

61. Sharabi Y, Sachs DH. Mixed chimerism and permanent specific transplantation tolerance induced by a nonlethal preparative regimen. J Exp Med 1989;169:493-502. [PubMed: 2562984]

62. Boros P, Bromberg JS. Human FOXP3+ regulatory T cells in transplantation. Am J Transplant 2009;9:1719-1724. [PubMed: 19538489]

63. Sakaguchi S, Yamaguchi T, Nomura T, Ono M. Regulatory T cells and immune tolerance. Cell 2008;133:775-787. [PubMed: 18510923]

64. Kingsley CI, Karim M, Bushell AR, Wood KJ. CD25+CD4+ regulatory T cells prevent graft rejection: CTLA-4- and IL-10- dependent immunoregulation of alloresponses. J Immunol 2002;168:10801086. [PubMed: 11801641] 
65. Cobbold S, Waldmann H. Infectious tolerance. Curr Opin Immunol 1998;10:518-524. [PubMed: 9794831]

66. Graca L, Cobbold SP, Waldmann H. Identification of regulatory T cells in tolerated allografts. J Exp Med 2002;195:1641-1646. [PubMed: 12070291]

67. Zheng XX, Sanchez-Fueyo A, Domenig C, Strom TB. The balance of deletion and regulation in allograft tolerance. Immunol Rev 2003;196:75-84. [PubMed: 14617199]

68. Sanchez-Fueyo A, Weber M, Domenig C, Strom TB, Zheng XX. Tracking the immunoregulatory mechanisms active during allograft tolerance. J Immunol 2002;168:2274-2281. [PubMed: 11859115]

69. Quezada SA, Fuller B, Jarvinen LZ, et al. Mechanisms of donor-specific transfusion tolerance: preemptive induction of clonal T-cell exhaustion via indirect presentation. Blood 2003;102:19201926. [PubMed: 12750162]

70. Jarvinen LZ, Blazar BR, Adeyi OA, Strom TB, Noelle RJ. CD154 on the surface of CD4+CD25+ regulatory $\mathrm{T}$ cells contributes to skin transplant tolerance. Transplantation 2003;76:1375-1379. [PubMed: 14627918]

71. Sanchez-Fueyo A, Tian J, Picarella D, et al. Tim-3 inhibits T helper type 1-mediated auto- and alloimmune responses and promotes immunological tolerance. Nat Immunol 2003;4:1093-1101. [PubMed: 14556005]

72. Yoshizawa A, Ito A, Li Y, et al. The roles of CD25+CD4+ regulatory T cells in operational tolerance after living donor liver transplantation. Transplant Proc 2005;37:37-39. [PubMed: 15808539]

73. Karim M, Feng G, Wood KJ, Bushell AR. CD25+CD4+ regulatory T cells generated by exposure to a model protein antigen prevent allograft rejection: antigen-specific reactivation in vivo is critical for bystander regulation. Blood 2005;105:4871-4877. [PubMed: 15713793]

74. Eljaafari A, Badet L, Kanitakis J, et al. Isolation of regulatory T cells in the skin of a human handallograft, up to six years posttransplantation. Transplantation 2006;82:1764-1768. [PubMed: 17198273]

75. Dijke IE, Caliskan K, Korevaar SS, et al. FOXP3 mRNA expression analysis in the peripheral blood and allograft of heart transplant patients. Transpl Immunol 2008;18:250-254. [PubMed: 18047933]

76. Muthukumar T, Dadhania D, Ding R, et al. Messenger RNA for FOXP3 in the urine of renal-allograft recipients. N Engl J Med 2005;353:2342-2351. [PubMed: 16319383]

77. Li XC, Demirci G, Ferrari-Lacraz S, et al. IL-15 and IL-2: a matter of life and death for T cells in vivo. Nat Med 2001;7:114-118. [PubMed: 11135625]

78. Lindahl KF, Wilson DB. Histocompatibility antigen-activated cytotoxic T lymphocytes. I. Estimates of the absolute frequency of killer cells generated in vitro. J Exp Med 1977;145:500-507. [PubMed: 233898]

79. Li XC, Strom TB, Turka LA, Wells AD. T cell death and transplantation tolerance. Immunity 2001;14:407-416. [PubMed: 11336686]

80. Kang HG, Zhang D, Degauque N, Mariat C, Alexopoulos S, Zheng XX. Effects of Cyclosporine on Transplant Tolerance: The Role of IL-2. Am J Transplant 2007;7:1907-1916. [PubMed: 17617853] 
Thymus

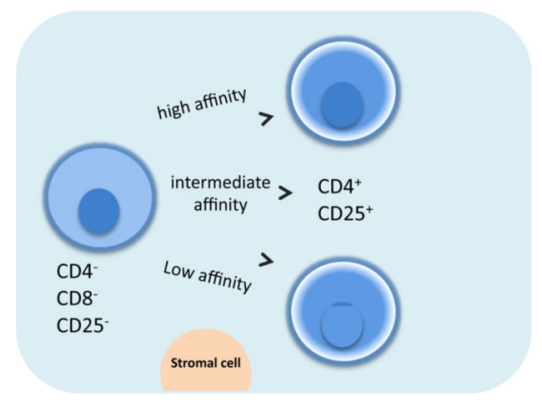

Periphery

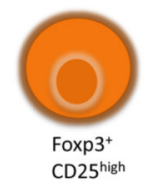

IL-2

Figure 1. CD4 ${ }^{+} \mathrm{CD25}^{+}$Treg Cell Development in the Thymus and Periphery

Development of CD4+ CD25+ Tregs's in the thymus involves interaction with thymic stromal cells via various molecules. An alternative model suggests clonal deletion as the predominant Treg developing mechanism within the thymus. In the periphery the majority of Treg's constitutively express high levels of Foxp3. The CD25 low Tregs's undergo homeostatic proliferation and/or Issue infiltration. Both CD25 high and CD25 low Treg's are immunosuppressive and express Foxp3. 


\section{Down regulation of allergic lungh inflammation}

\section{Suppression of in vitro induced antigen- and mitogen-driven T-cell proliferation}

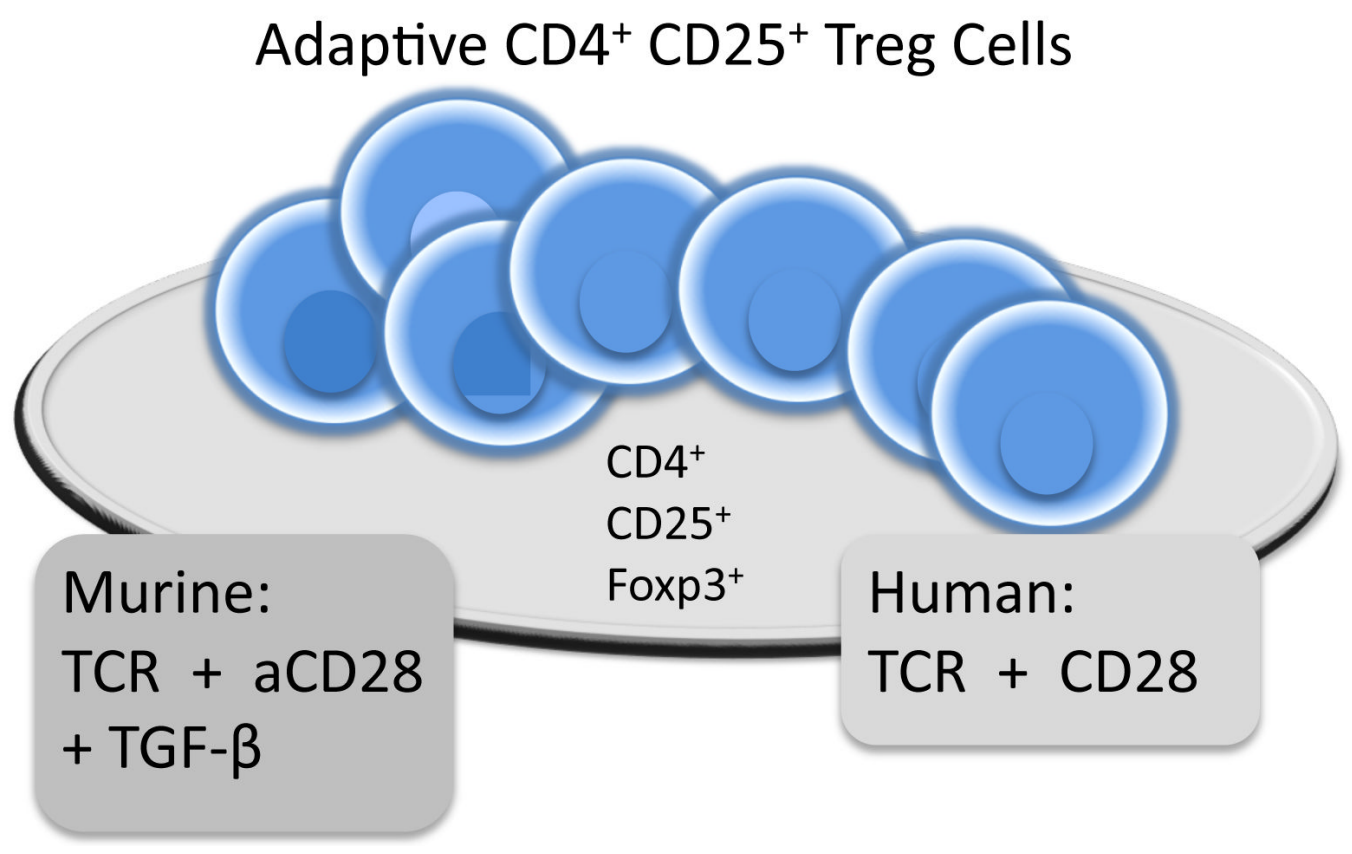

Figure 2. AdapIve (Inducible) $\mathrm{CD}^{+} \mathrm{CD}^{+} 5^{+}$Treg Cells

Murine adaptive CD4+ CD25+ Treg's can be derived in vitro through treatment of peripheral CD4+ CD25+ Treg's with TCR, anti CD28 antibodies and TGF- $\beta$. Human adaptive CD4+ CD25+ Treg's can be induced in the absence of TGF- $\beta$. Both murine and human adaptive Treg's express Foxp3 are capable to down rgulate allergic lungh inflammation and suppress in vitro induced antigen and mitogen driven $\mathrm{T}$ cell proliferation. 


\section{Inhibition of allo- and autoimmune responses}
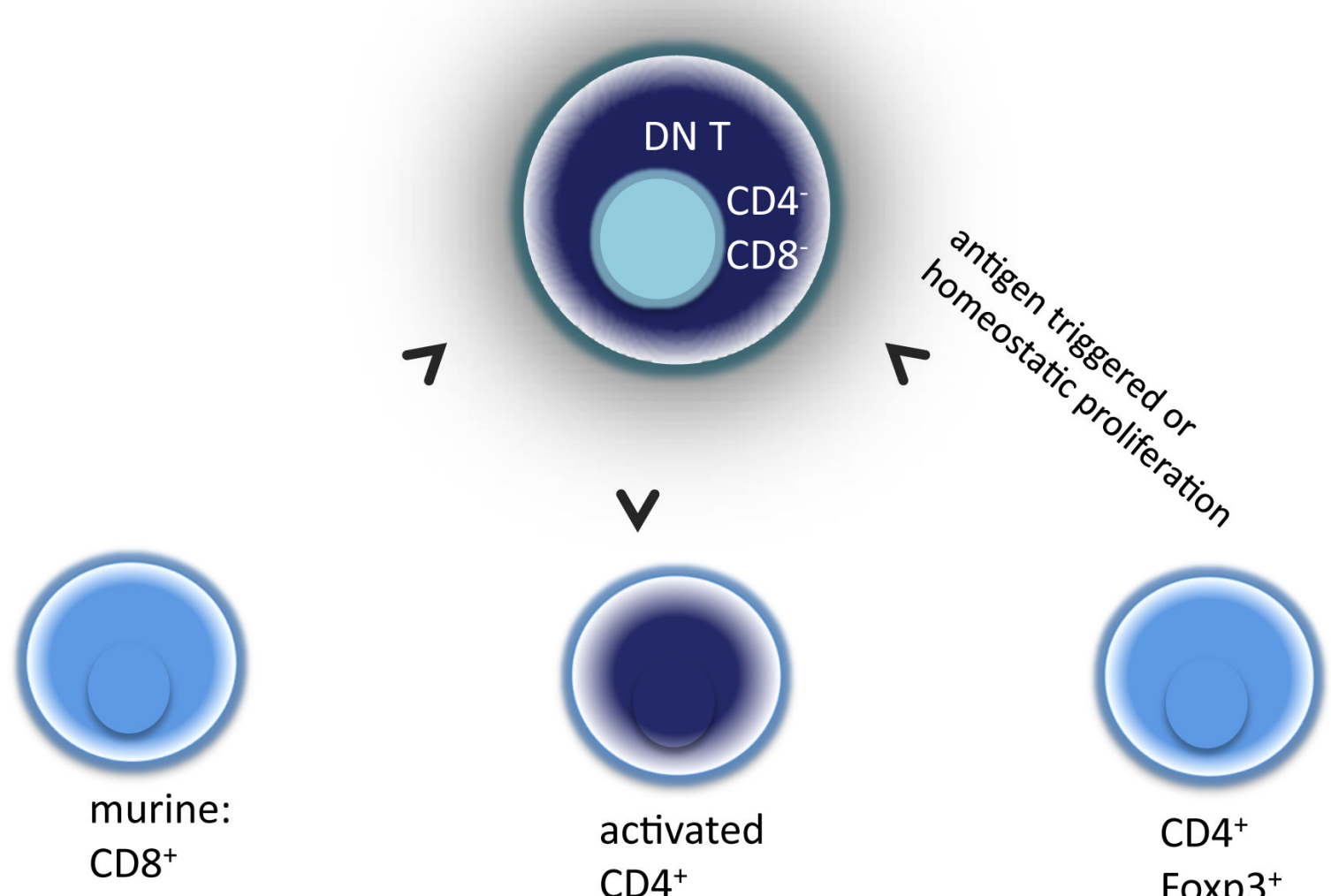

$\mathrm{CD}^{+}$

Foxp3 $^{+}$

\section{CD25-}

Figure 3. CD4-CD8- (double-negatve, DN) T-cells

Although only $1-5 \%$ in periperal lymphoid Issues, DN Treg's are potent in inhibiting allo und autoimmune responses in an antigen specific manner. In murine models DNT can be derived directly from CD8+ T cells, or arise extrathymicly from bone marrow. 


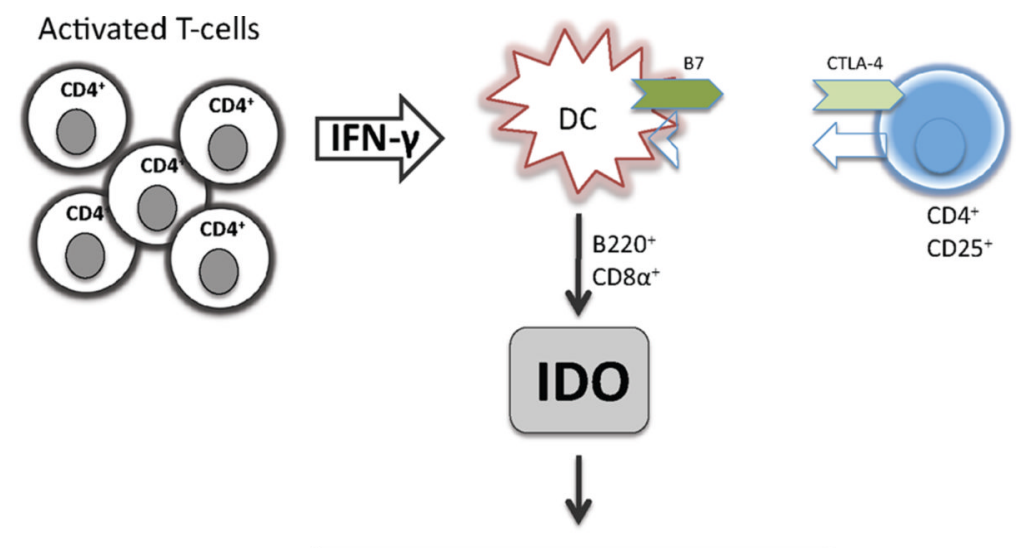

Control of immune respronse

Promotion of systemic tolerance

Figure 4. Indoleamine 2,3-dioxygenase (IDO) and Tregs

Crosslinking of CTLA-4 on regulatory T cells (Tregs) and B7 on dendritic cells (DC) leads to the induction of IDO and IFN- $\gamma$ on DCs and B220+ CD8 $\alpha+$ DCs. This resulting immunomodulatory enviroment is capable to suppress local $\mathrm{T}$ cell responses and promotes systemic tolerance. 